\title{
Loss of PALB2 predicts poor prognosis in acute myeloid leukemia and suggests novel therapeutic strategies targeting the DNA repair pathway
}

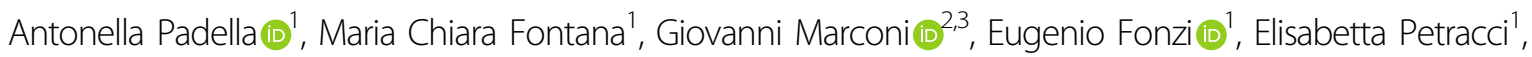 \\ Anna Ferrari (1)', Carmen Baldazzi ${ }^{2,3}$, Cristina Papayannidis ${ }^{2,3}$, Andrea Ghelli Luserna Di Rorá', Nicoletta Testoni ${ }^{2,3}$, \\ Gastone Castellani ${ }^{2,3}$, Torsten Haferlach ${ }^{4}$, Giovanni Martinelli (10) and Giorgia Simonetti (1)
}

Dear Editor,

Acute myeloid leukemia (AML) patients carrying complex karyotype or aneuploidies have a very poor prognosis, with a 5 -year overall survival (OS) $<20 \%{ }^{1}$. We and others have shown that these patients are characterized by high genomic instability, along with defects of DNA damage response (DDR) genes ${ }^{2,3}$.

Partner and localizer of BRCA2 (PALB2) is a key player in the homology recombination (HR) pathway, since it functions as a scaffold for the HR complex ${ }^{4}$. PALB2 monoallelic germline mutations are associated with an increased risk of developing breast, pancreatic, and ovarian cancers, whereas biallelic mutations lead to Fanconi anemia ${ }^{5}$. Genomic lesions, including sequence mutations and copy-number alterations (CNAs) of HR and DDR genes, are rare events in sporadic cancers and no recurrent genomic alterations in these genes have been reported so far in AML.

Here, we studied the genomic alterations of PALB2 in AML, including its molecular interactions and prognostic relevance.

Single-nucleotide polymorphism (SNP) arrays of 119 adult AML cases and whole-exome sequencing (WES) data of 68 adult AML cases were downloaded from the NGSPTL project repository. Mutational data from the BeatAML study $(n=531)$ and acute erythroid leukemia (AEL)

\footnotetext{
Correspondence: Antonella Padella (antonella.padella@irst.emr.it) or Giovanni Martinelli (giovanni.martinelli@irst.emr.it)

${ }^{1}$ Istituto Scientifico Romagnolo per lo Studio e Cura dei Tumori (IRST) IRCCS, Meldola, Italy

${ }^{2}$ Azienda Ospedaliero-Universitaria di Bologna, via Albertoni 15, Bologna, Italia, Istituto di Ematologia "Seràgnoli", Dipartimento di Medicina Specialistica, Diagnostica e Sperimentale, Università degli Studi, Bologna, Italia Full list of author information is available at the end of the article
}

cohort $(n=159)$ were retrieved from https://www. cbioportal.org/ and https://pecan.stjude.cloud/, respectively. The GSE23452 SNP array $(n=144)$, the GSE14468 $(n=486)$, and The Cancer Genome Atlas (TCGA, $n=200)$ AML transcriptomic datasets were obtained from Gene Expression Omnibus collection (https://www.ncbi.nlm.nih. gov/gds) and https://cancergenome.nih.gov/, respectively. SNP array data files for the NGS-PTL cohort $(n=119)$ are available through the Gene Expression Omnibus public database (accession no. GSE160982). Detailed dataset information and analysis are described in the Supplemental Material section.

We first looked for PALB2 sequence variations in publicly available genomic datasets (TCGA, Beat-AML, NGS-PTL, and AEL): one somatic frameshift mutation was identified in one AML patient (NM_024675:exon3: T51Qfs*2, TCGA cohort) and two missense mutations (NM_024675:exon 4:Q460R, NM_024675:exon 4:G165C) were identified in two AEL cases. We then asked whether PALB2 was affected by CNAs. PALB2 CN loss was detected in $12 / 233$ patients (5.2\%), with a minimal common deleted region of $6.6 \mathrm{~kb}$ at the $\mathrm{C}$ terminus. This region included exon 12, which encodes for the domain responsible for the interaction with the $H R$ proteins RAD51 and BRCA2 and the DNA repair-related polymerase POLH (Fig. 1A and Supplementary Table 1). PALB2 deletion was associated with $\mathrm{CN}$ loss of genomic regions frequently altered in poor-prognosis AML, including chromosome $5 \mathrm{q}$ and $17 \mathrm{p} 13(p<0.05$ and false discovery rate $<0.25$; Fig. $1 \mathrm{~B}$ and Supplementary Table 2 ). AML patients carrying PALB2 genomic loss were older than wild-type cases (median age of 65 years vs. 59 in the wild-type cohort, $p=0.044$; Table 1 ) and were 


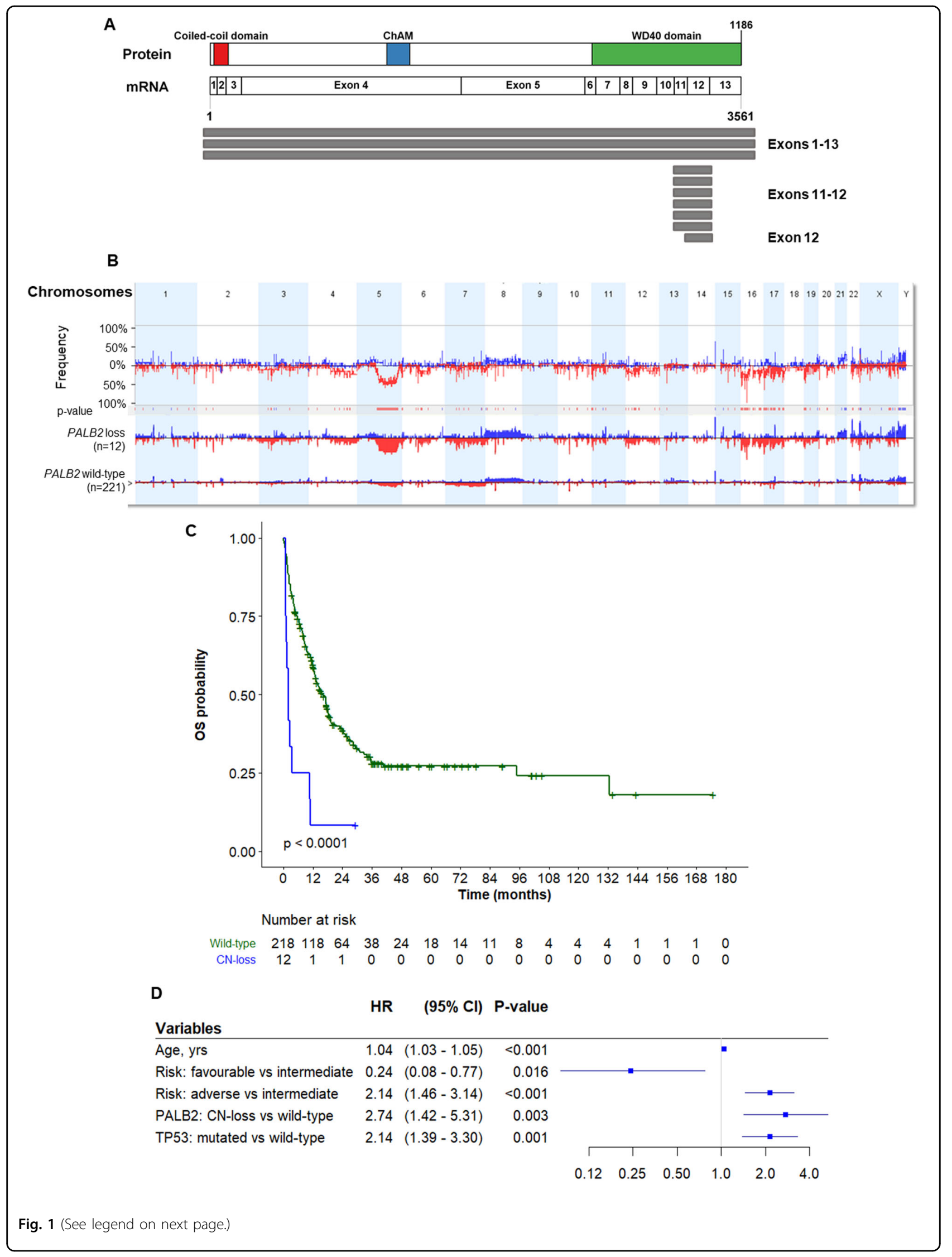


(see figure on previous page)

Fig. 1 PALB2 CN loss in AML and its prognostic relevance. A Graphical representation of PALB2 mRNA and protein functional domains. The coiled-coil domain (red) is involved in the interaction with BRCA1; the chromatin-association motif (ChAM, blue) is required for chromatin association and it mediates nucleosome association; the WD40 domain (green) is responsible for the interaction with RAD51, BRCA2, and POLH. Gray bars represent CN loss detected in AML patients. B Frequency (\%) and the significance of genomic CN changes between PALB2 loss and wild-type patients. Blue indicates CN gains and red indicates CN loss. Results are summarized for a group of patients (PALB2 loss vs. PALB2 wild-type). C Kaplan-Meier curve of PALB2 loss (blue) vs. PALB2 wild-type (green) AML. D Forest plot for the multivariable Cox regression analysis. yrs years, HR hazard ratio, $\mathrm{Cl}$ confidence interval.

Table 1 Comparison of biological and molecular features between PALB2 loss and PALB2 wild-type AML cases.

\begin{tabular}{|c|c|c|c|}
\hline & $\begin{array}{l}\text { PALB2 wt } \\
(n=221)\end{array}$ & $\begin{array}{l}\text { PALB2 CN loss } \\
(n=12)\end{array}$ & $P$ value \\
\hline Gender, $n$ (\%) & & & 1 \\
\hline Female & $89(40.3)$ & $5(41.7)$ & \\
\hline Male & $132(59.7)$ & 7 (58.37) & \\
\hline Age (years) & & & 0.044 \\
\hline Median [min-max] & 59.2 [18.1-88.5] & 65.3 [46.6-85.9] & \\
\hline NA & 1 & - & \\
\hline Type of AML, n (\%) & & & 0.880 \\
\hline De novo & $163(73.8)$ & $10(83.4)$ & \\
\hline Secondary to MN & $38(17.2)$ & $1(8.3)$ & \\
\hline $\mathrm{t}-\mathrm{AML}$ & $20(9.0)$ & $1(8.3)$ & \\
\hline Risk, n (\%) & & & 0.016 \\
\hline Favorable & $15(6.8)$ & $1(8.3)$ & \\
\hline Intermediate & $124(56.1)$ & $2(16.7)$ & \\
\hline Adverse & $82(37.1)$ & $9(75.0)$ & \\
\hline TP53, n (\%) & & & 0.001 \\
\hline wt & $177(80.4)$ & $4(33.3)$ & \\
\hline Mutated & 43 (19.6) & $8(66.7)$ & \\
\hline NPM1, n (\%) & & & 0.370 \\
\hline wt & $172(85.1)$ & $11(100.0)$ & \\
\hline Mutated & $30(14.9)$ & $0(0.0)$ & \\
\hline NA & 19 & 1 & \\
\hline FLT3-ITD, n (\%) & & & 1 \\
\hline wt & $182(87.1)$ & $11(91.7)$ & \\
\hline Mutated & $27(12.9)$ & $1(8.3)$ & \\
\hline NA & 12 & - & \\
\hline
\end{tabular}

wt Wild type, $C N$ copy number, $M N$ myeloproliferative neoplasms, NA not available, $t-A M L$ therapy-related AML.

characterized by a poor prognosis $(75.0 \%$ vs. $37.1 \%$ of PALB2 wild-type cases, $p=0.016$; Table 1 ).

To better understand the phenotype of AML carrying PALB2 CN loss, we performed pathway analysis of genes significantly affected by CNAs in those patients (compared with PALB2 wild-type cases, $p<0.05)$. WNT and ERBB2 signaling pathways, cell adhesion, cytokines, and inflammatory response, metabolism, necroptotic process, senescence, and autophagy in cancer were the most relevant pathways enriched of genes affected by $\mathrm{CN}$ loss $(p<0.01$, adj- $p<0.25$; Supplementary Table 3$)$. By integrating $\mathrm{CN}$ and mutational data, we observed an association between PALB2 deletions and TP53 genomic alterations: 8/12 PALB2 loss patients had TP53 somatic mutations (66.7\%), in comparison to $19.6 \%$ of $P A L B 2$ wild-type cases $(p<0.001$; Table 1$)$. Six patients with PALB2 loss and TP53 mutations had biallelic inactivation of TP53 (loss of heterozygosity: $n=1$; CN loss: $n=5$ ), indicating that TP53 functionality was potentially lost in these cases. To identify additional mutations that cooperated with PALB2 loss, we integrated $\mathrm{CN}$ results with WES data $(n=68)$ of available AML cases from the NGSPTL cohort ${ }^{3}$ that was enriched for patients having complex karyotype (Supplementary Table 4). In this cohort, 6 out of 68 patients $(8.8 \%)$ carried PALB2 CN loss. In addition to TP53 $(n=3,50.0 \%), P A L B 2$ loss patients had mutations in DNMT3A $(n=2,33.3 \%), B C O R(n=1)$, CDKN2A $(n=1)$, FLT3 $(n=1), N P M 1(n=1), N R A S$ $(n=1)$, and $\operatorname{WT1}(n=1,16.6 \%$; Supplementary Fig. 1$)$.

It has been previously reported that heterozygous PALB2 genomic alterations, including deletion and truncating or single-nucleotide variants induced a reduction of protein expression $^{6,7}$. Here, we analyzed PALB2 expression in leukemic cells and its potential association with patients' biological or molecular features. Data from two independent cohorts revealed that elderly patients expressed lower levels of PALB2 than younger ones (TCGA: PALB2 median expression 21.3 vs. 23.3 counts per million, respectively, $p=0.007$; GSE14468: PALB2 median expression 213.8 vs. 232.3, respectively, $p=0.055$; Supplementary Table 5). This result is in line with the above data on AML patients carrying PALB2 genomic loss. Notably, we observed significant differences in PALB2 levels among cytogenetic risk classes in the two cohorts (GSE14468, $p=0.020$ and TCGA, $p=0.006)$. Specifically, patients with favorable prognosis $(\mathrm{t}(8 ; 21)$ or inv(16) AML) expressed higher levels of PALB2, while intermediate and adverse risk cases had lower expression (normal karyotype, complex karyotypes, KMT2A-rearranged AML, and other abnormalities; 
Supplementary Table 5). This observation also recapitulates the association between $P A L B 2$ genomic loss and high cytogenetic risk. The association between PALB2 expression and karyotype was not confirmed in the GSE14468 dataset, potentially due to the unbalanced proportion of cases belonging to the different cytogenetic subgroups between the two cohorts.

We then investigated the prognostic role of genetic alterations of $P A L B 2$ in AML. Despite the low number of altered cases, $P A L B 2$ loss patients had a worse prognosis compared to PALB2 wild-type ones (median OS PALB2 loss: 2.0 months, $95 \%$ confidence intervals (CIs): $1.2-$ not reached; PALB2 wild-type: 16.2 months, 95\% CIs: 12.9-19.1; $p<0.0001$; Fig. 1C). Moreover, in a multivariate Cox model PALB2 loss negatively affected the prognosis of AML patients with a hazard ratio of 2.74 (95\% CIs: $1.42-5.31, p=0.003$; Fig. 1D) and its effect was not dependent on age, cytogenetic risk, and, notably, on TP53 mutational status (which were significant predictors in univariate analysis and were in most cases associated with PALB2 loss). Therefore, PALB2 might be a novel biomarker of poor prognosis in AML.

$P A L B 2$ is a tumor suppressor affected by mutations and CNAs in patients with either a familiar history of breast, pancreatic, and ovarian cancers ${ }^{5}$, or, rarely, in sporadic solid and hematological tumors (https://www.cbioportal. org/). Notably, PALB2 participates also in the Fanconi anemia pathway and biallelic germline mutations were associated with the development of AML, among other childhood cancers ${ }^{4}$.

In this study, we report that PALB2 is rarely mutated at the somatic level in adult AML. However, in a cohort of 233 patients, PALB2 CN loss was detected in $5.2 \%$ of cases and it was associated with TP53 genomic alterations. Large $P A L B 2$ genomic deletions were also reported in one AML, one myelodysplastic syndrome ${ }^{8}$, and four AEL cases 9 Remarkably, the co-association of PALB2 and TP53 alterations has been previously described in PALB2-related breast cancer ${ }^{10}$. Moreover, in our cohort, we detected seven patients with a focal deletion involving exons 11 and 12 , which encode for the WD40 domain. Mutations in this domain were reported to disrupt DNA repair activity ${ }^{6}$.

Enrichment analysis of genes within chromosomal regions affected by $\mathrm{CN}$ loss and significantly associated with PALB2 loss pointed to the ERBB2 (HER2) signaling pathway (5/37 enriched pathways, $p \leq 0.0114)$. These data suggest that the phenotype of PALB2 loss patients might resemble that of HER2-negative breast cancers. Of note, breast cancer patients carrying PALB2 germline mutations were associated with the HER2-negative phenotype $^{10}$. No familial history of cancer was reported in patients from our AML cohort, suggesting that the detected PALB2 CN loss might be somatic alterations acquired by the leukemic cells. However, we cannot exclude the germline origin of the deletion, since pairedgermline samples were not available.

Few studies described an association between PALB2 genomic lesions and patients' outcome so far ${ }^{11}$. We here show a negative impact of $P A L B 2$ genomic alterations on AML patients' survival with cases carrying PALB2 loss having shorter OS. This predictive role was independent of other negative prognostic factors (e.g., age, cytogenetic risk, and, notably, TP53 alterations). Nevertheless, a limitation of our study is the heterogeneity of treatments and patients' age, pointing out the need for a larger and homogeneous AML cohort to validate the potential of $P A L B 2$ as a biomarker for poor-prognosis AML.

At the therapeutic level, high PALB2 mRNA levels predicted response to cisplatin-docetaxel in non-small cell lung cancer ${ }^{12}$, while PALB2-mutated pancreatic cancers were described to benefit from mitomycin $C$ or PARP inhibition (PARPi) treatment ${ }^{8,13}$. Despite the potential relevance, these studies were characterized by a limited number of patients and the role of PALB2 alterations in therapy response deserves further investigation. This is particularly important in AML, where functional studies elucidating PALB2 role in AML cells are needed. PARPi is currently being explored as therapeutic options alone or in combination with chemotherapy, alkylating, or demethylating agents in $\mathrm{AML}^{2}$. Different studies reported a deregulated expression of HR genes in AML and preclinical studies showed that $\mathrm{t}(8 ; 21)$ and $\mathrm{t}(15 ; 17)$ AML are extremely sensitive to $\mathrm{PARPi}^{2,14,15}$. Taken together, our data indicate that therapies targeting the HR pathway may become a valuable therapeutic option for the subgroup of AML patients characterized by PALB2 $\mathrm{CN}$ loss and dismal outcome.

\section{Acknowledgements \\ This project was supported by the Torsten Haferlach Leukämiediagnostik Stiftung, the Italian Ministry of Health (IT-MoH) [Italian Ministry of Health Current Research 2018], and under the frame of ERA PerMed (ERA Net Grant 779282).}

\begin{abstract}
Author details
${ }^{1}$ Istituto Scientifico Romagnolo per lo Studio e Cura dei Tumori (IRST) IRCCS, Meldola, Italy. ${ }^{2}$ Azienda Ospedaliero-Universitaria di Bologna, via Albertoni 15, Bologna, Italia, Istituto di Ematologia "Seràgnoli", Dipartimento di Medicina Specialistica, Diagnostica e Sperimentale, Università degli Studi, Bologna, Italia. ${ }^{3}$ Dipartimento di Medicina Specialistica, Diagnostica e Sperimentale, Istituto di Ematologia "Seràgnoli", Università degli Studi, Bologna, Italy. ${ }^{4}$ MLL Munich
\end{abstract} Leukemia Laboratory, Munich, Germany

Conflict of interest

T.H. is part owner of MLL Munich Leukemia Laboratory.

\section{Publisher's note}

Springer Nature remains neutral with regard to jurisdictional claims in published maps and institutional affiliations.

Supplementary Information accompanies this paper at (https://doi.org/ 10.1038/s41408-020-00396-x). 
Received: 17 September 2020 Revised: 16 November 2020 Accepted: 27 November 2020

Published online: 07 January 2021

\section{References}

1. Papaemmanuil, E. et al. Genomic classification and prognosis in acute myeloid leukemia. N. Engl. J. Med. 374, 2209-2221 (2016).

2. Faraoni, I., Giansanti, M., Voso, M. T., Lo-Coco, F. \& Graziani, G. Targeting ADPribosylation by PARP inhibitors in acute myeloid leukaemia and related disorders. Biochem. Pharmacol. 167, 133-148 (2019).

3. Simonetti, G. et al. Aneuploid acute myeloid leukemia exhibits a signature of genomic alterations in the cell cycle and protein degradation machinery. Cancer https://doi.org/10.1002/cncr.31837 (2018).

4. Ducy, M. et al. The tumor suppressor PALB2: inside out. Trends Biochem. Sci. 44 226-240 (2019).

5. Nepomuceno, T. et al. The role of PALB2 in the DNA damage response and cancer predisposition. Int. J. Mol. Sci. 18, 1886 (2017).

6. Boonen, R. A. C. M. et al. Functional analysis of genetic variants in the high-risk breast cancer susceptibility gene PALB2. Nat. Commun. 10, 1-15 (2019).

7. Nikkilä, J. et al. Heterozygous mutations in PALB2 cause DNA replication and damage response defects. Nat. Commun. 4, 1-8 (2013).
8. Bhangoo, M. S. et al. Biallelic deletion of PALB2 occurs across multiple tumor types and suggests responsiveness to poly (ADP-ribose) polymerase inhibition. JCO Precis. Oncol. https://doi.org/10.1200/po.17.00043, 1-7 (2017).

9. lacobucci, I. et al. Genomic subtyping and therapeutic targeting of acute erythroleukemia. Nat. Genet. 51, 694-704 (2019).

10. Li, A. et al. Homologous recombination DNA repair defects in PALB2associated breast cancers. npj Breast Cancer 5, 1-14 (2019).

11. Cybulski, C. et al. Clinical outcomes in women with breast cancer and a PALB2 mutation: a prospective cohort analysis. Lancet Oncol. 16, 638-644 (2015).

12. Karachaliou, N. et al. Association of PALB2 messenger RNA expression with platinum-docetaxel efficacy in advanced non-small cell lung cancer. J. Thorac. Oncol. 14, 304-310 (2019).

13. Villarroel, M. C. et al. Personalizing cancer treatment in the age of global genomic analyses: PALB2 gene mutations and the response to DNA damaging agents in pancreatic cancer. Mol. Cancer Ther. 10, 3-8 (2011).

14. Nieborowska-Skorska, M. et al. Gene expression and mutation-guided synthetic lethality eradicates proliferating and quiescent leukemia cells. J. Clin. Invest. 127, 2392-2406 (2017).

15. Nieborowska-Skorska, M. et al. Inhibition of the mutated c-KIT kinase in AML1ETO-positive leukemia cells restores sensitivity to PARP inhibitor. Blood Adv. $\mathbf{3}$ 4050-4054 (2019). 